\title{
The re-awakening of $\mathrm{hCG}$ expression. Its role in the diagnosis of cervical squamous cell carcinoma
}

\author{
Zehra Bozdag, MD, Aynur M. Taslar, MD, Neslihan B. Tepe, MD, Omer F. Dizibuyuk, MD, Huseyin C. Ozcan, MD,
} Metin Karakök, MD.

\begin{abstract}

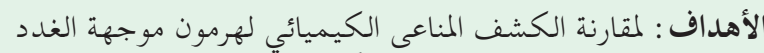

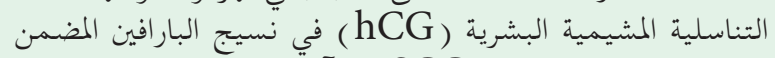

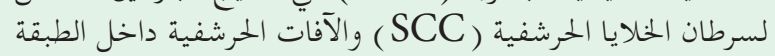
الرفيعة (HSIL)

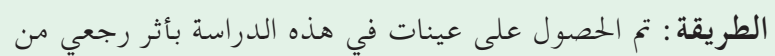

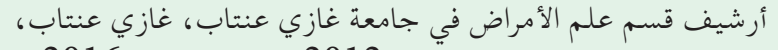

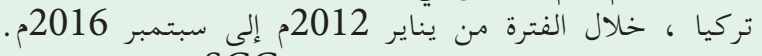

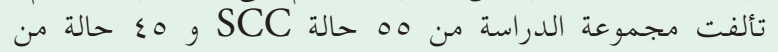
HSIL

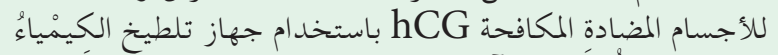

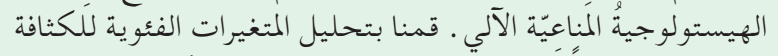

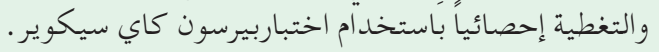

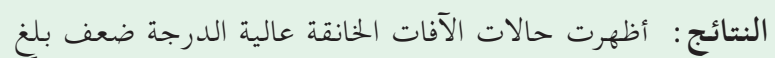

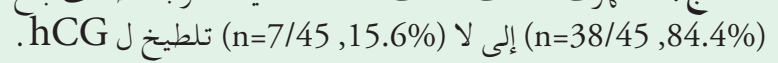

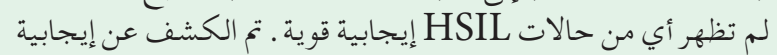

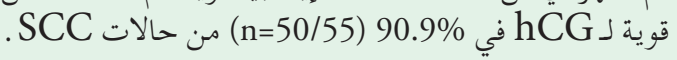

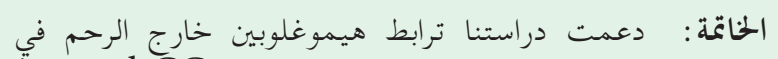

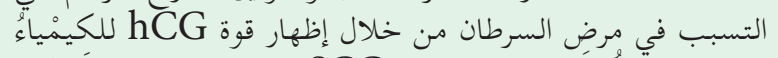

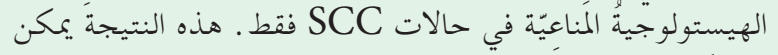

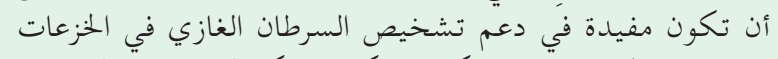

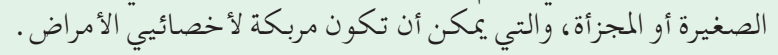

Objectives: To compare immunohistochemical detection of Human chorionic gonadotropin (hCG) expression in paraffin embedded tissue of squamous cell carcinomas (SCC) and high grade squamous intraepithelial lesions (HSIL).

Methods: The samples in this retrospective study were obtained from the archives of the Pathology Department at Gaziantep University, Gaziantep, Turkey, over the period from January 2012 to September 2016. The study group consisted of 55 cases of SCC and 45 cases of HSIL. Tissue expression of hCG was detected by specific binding of anti-hCG antibody using an automated immunohistochemistry staining device. The categorical variables of intensity and coverage were analyzed statistically using Pearson Chi-Square test.

Results: High grade squamous cell lesions cases showed weak $(84.4 \%, \mathrm{n}=38 / 45)$ to no $(15.6 \%$, $\mathrm{n}=7 / 45)$ staining for hCG. None of the HSIL cases showed strong positivity. Strong positivity for hCG was detected in $90.9 \%(n=50 / 55)$ of SCC cases.

Conclusion: Our study supports the association of ectopic hCG expression in cancer pathogenesis by demonstrating strong hCG immunoreactivity only in SCC cases. This finding can be helpful in supporting the diagnosis of invasive carcinoma in small or fragmented biopsies, which can on their own be confusing for the pathologists.

Saudi Med J 2018; Vol. 39 (9): 873-877 doi: $10.15537 /$ smj.2018.9.22710

From the Department of Pathology (Bozdag, Dizibuyuk, Karakök), and from the Department of Obstetrics and Gynecology (Taslar, Tepe, Ozcan), Medical School, Gaziantep University, Gaziantep, Turkey.

Received 14th May 2018. Accepted 25th July 2018.

Address correspondence and reprint request to: Dr. Aynur M. Taslar, Department of Obstetrics and Gynecology, Medical School, Gaziantep University, Gaziantep, Turkey. E-mail:dr.aynur.moustafa@hotmail.com ORCID ID: orcid.org/0000-0003-1071-1976

O ervical cancer is the third most common cancer in women worldwide and the second most common cancer in developing countries. The importance of this cancer depends on its high prevalence and young average age of death. ${ }^{1}$ It is well known that persistent infection with oncogenic human papillomavirus (HPV) contributes to the majority of cervical SCC cases. 
The major steps in cervical cancer development are: infection of the metaplastic epithelium at the squamocolumnar junction, viral persistence, progression to cervical precancerous lesion, and then invasion through the basement membrane of epithelium. However, the progression of a preinvasive lesion to an invasive carcinoma is still an enigma. ${ }^{2}$

Human chorionic gonadotropin has been known for decades as pregnancy related hormone that can help clinicians in early detection of pregnancy, it acts as a tumor marker in gestational trophoblastic tumors as well. Human chorionic gonadotropin is a glycoprotein hormone consisting of $\alpha$ and $\beta$ subunit which has a unique structure consisting of 5 separate molecules with independent functions. ${ }^{3,4}$ These are: hCG, sulfated hCG, hyperglycosylated hCG, hCG free ß-subunit and hyperglycosylated hCG free $ß$-subunits. Both of hyperglycosylated hCG and hCG free $ß$-subunit can antagonize the Transforming growth factor beta (TGFß) receptor and are thus considered as oncogenisis factors. ${ }^{5-7}$ This function has been proved by considerable researches that demonstrated hCG expression in many different malignancies such as bladder, cervix, endometrium, pancreas, lung and vulva besides choriocarcinoma, gestational trophoblastic tumors, ovarian, and testicular germ cell tumors. ${ }^{3,6}$ Human chorionic gonadotropin is inferred as a marker of invasion in gynecologic and non-gynecologic tumors. ${ }^{5,6}$

In this study, we aimed to investigate the expression of hCG in SCC, and HSIL to detect the possible role of this expression in the diagnosis of SCC.

Methods. Patientsandsamplescollection. Thesamples included in this retrospective study were obtained from the archives of the Pathology Department, at Gaziantep University, Faculty of Medicine, Gaziantep, Turkey, over the period from January 2012 to September 2016. The study group consisted of 55 cases of SCC and 45 cases of HSIL.

Inclusion criteria. It was specimens of SCC derived from patients who had undergone total hysterectomy, and HSIL specimens were derived from patients who had undergone the loop electrosurgical excision procedure (LEEP) or cone biopsy.

Exclusion criterion. cases containing a limited

Disclosure. Authors have no conflict of interests, and the work was not supported or funded by any drug company. amount of intraepithelial lesion or tumor tissues. The clinical data were retrieved from the hospital records. Ethical Committee's approval according to the principles of Helsinki declaration was awarded from this study $(16 / 235)$.

Immunohistochemical staining. The Hematoxylin Eosin (H\&E) and immunohistochemistry stained slides of each case were re-examined under light microscopy by pathologists. Four-micron sections were taken from selected formalin-fixed-paraffin-embedded tissue blocks. The Immunohistochemical hCG antibody (rabbit polyclonal antibody, ready to use, Roche ${ }^{\oplus}$, Catalog number: 760-2650, USA) was studied using an automated immunohistochemistry-staining device (Ventana, BenchMark Ultra Auto-Stainer, USA). A tissue section of normal placenta showed positive cytoplasmic staining, used as positive control.

The immunohistochemical results were scored according to the published criteria. ${ }^{8}$ The immunoreactivity of the cases for hCG were graded based on 2 parameters: the percentage of positive cells and the intensity of immunopositivity (at x200 magnification). The numerical scores for the percentage of immunopositivity were: 0 (as negative), 1 (positivity in $1-10 \%$ of tumour cells), 2 (positivity in $11-50 \%$ of tumour cells) and 3 (positivity in $>50 \%$ of tumour cells). The intensity of staining was scored as none $(0)$, mild $(1+)$, moderate $(2+)$ or strong $(3+)$. The intensity and percentage scores assigned to each lesion were multiplied to give a final histo score (H-score). The range of possible scores were $0-12$.

Statistical analysis. The categorical variables were analyzed by using Pearson Chi-Square test with SPSS ${ }^{\odot}$ Version 23.0 (IBM Corp., Armonk, NY, USA). A $p$-value $<0.05$ was considered significant.

Results. In this study, we investigated staining intensity and percentage in both HSIL and SCC cases. We examined 45 conization materials of HSIL cases and the normal squamous epithelium (NSE) nearby this area.

Fourty five of HSIL cases were examined, $84.4 \%$ of HSIL ( $\mathrm{n}=38 / 45)$ showed diffuse weak positivity, while $15.6 \%(n=7)$ of them showed no immune reactivity for hCG. Normal squamous epithelium showed weak to no staining as did HSIL, 66\% ( $\mathrm{n}=30)$ of NSE showed weak staining, and $34 \%(n=15)$ of them showed no staining.

Fifty-five hysterectomy materials of cervical SCC specimens were examined. Strong positivity for hCG (Figure 1) was detected in $90.9 \%(n=50 / 55)$ of cases, whereas $4.9 \%(n=5 / 55)$ of cases showed no staining for 
hCG. The data is summarized in (Table 1). The intensity of positivity was markedly strongest at the periphery of the tumor islands.

There is a statistically significant correlation between the intensity of staining in SCC and HSIL cases ( $p$-value $=0.001)$.

Discussion. There are varieties of malignancies that can produce hCG during their growth and in which hCG expression has been associated with the aggressive behavior of these tumors. Hyperglycosylated hCG, hCG $\beta$ and hyperglycosylated hCG $\beta$ have been proposed to be interchangeable cancer promoters. ${ }^{3}$ However, to have better understanding of this ectopic expression, much more research is needed concerning $\beta$ hCG molecular structure and in particular the forms expressed by cancer. ${ }^{9,10}$
Human chorionic gonadotropin is a glycoprotein hormone that is considered to be the most acidic and glycosylated protein in human body. It consists of an $\alpha$ and $\beta$ subunit but the combination and variants that have been proposed to encompass 5 separate molecules with independent functions: sulfated hCG (LH hormone mimicking) secreted from pituitary gland which fosters follicles and also induces ovulation and luteogenesis, hCG free $\beta$-subunit (TGFß receptor antagonist) inhibits apoptosis and stimulates cancer-like process, hyperglycosylated hCG secreted from cytotrophoblast, which promotes placental implantation and growth and also responsible for choriocarcinoma invasion by suppressing apoptosis, and hCG derived from syncytiotrophoblast, which induces progesterone production from corpus luteum, stimulates fetal and umbilical development, increases uterine growth and

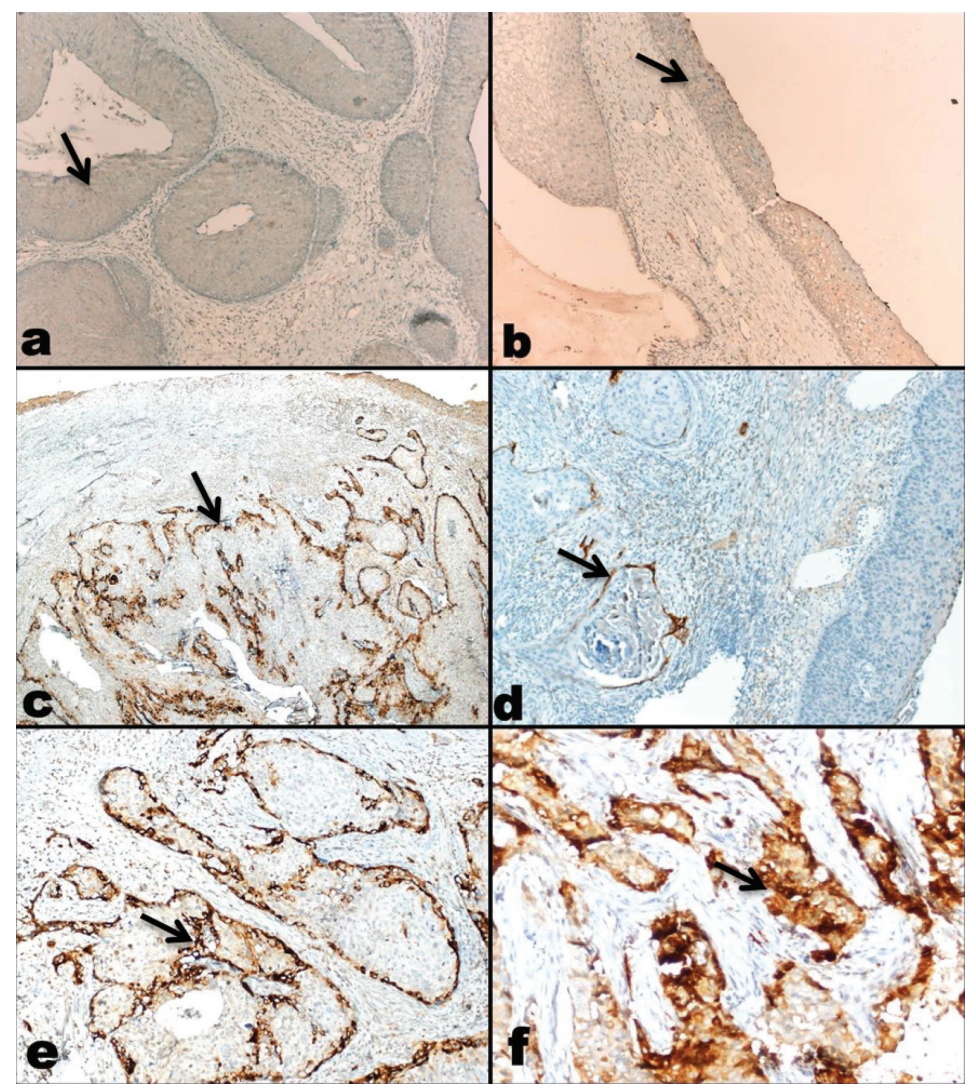

Figure 1 - IIllustration of immunohistochemical staining for hCG, demonstrating strong expression is related to squamous cell carcinoma (SCC). Panel a) weak immunohistochemical staining in high grade squamous intraepithelial lesions (HSIL) for human chorionic gonadotropin (hCG) (x100 photomicrograph); Panel b) weak immunohistochemical staining for hCG expression in high grade squamous intraepithelial lesions HSIL and adjacent NSE (x100 photomicrograph); Panel c) strong immunohistochemical staining for hCG expression in SCC, prominent at the periphery of the tumor (x40 photomicrograph); Panel d) strong, score 1 (1-10\% of tumor cells) immunohistochemical staining for hCG expression (x100 photomicrograph); Panel e) strong, score 2 (50\% of tumor cells) immunohistochemical staining for hCG expression (x200 photomicrograph); Panel f) strong, score 3 ( $\geq 50 \%$ of tumor cells) immunohistochemical staining for hCG (x200 photomicrograph). 
angiogenesis and may trigger nausea and vomiting during pregnancy. Generally, whole hCG molecule and hCG free $\beta$-subunit are measured in pregnant women sera. ${ }^{3,11}$

Both of the hyperglycosylated hCG and hCG free $\beta$-subunit can antagonize TGF $\beta$ receptor (acting as autocrine hormone), and can thus be categorized as oncogenisis factors. ${ }^{4}$ However, cancers can be divided into 2 types depending on their hCG variant. On one hand, hyperglcosylated hCG promotes cell invasion in some tumors like; gestational trophoplastic disease, choriocarcinoma, and germ cell tumors. On the other hand, hCG free $\beta$-subunit can be expressed by variety malignancies such as lung cancer, breast cancer, leukemia, lymphoma, cervical cancer and so on. ${ }^{4,12}$

Although hCG can be measured in both serum and urine specimens, urinary gonadotropin fragment (UGF) is detected solely in urine samples that may explained by the high metabolic clearance rate of hCG free $\beta$-subunit. ${ }^{3}$ This can be observed vividly in patients at third stage cervical cancer where the impairment kidney function will lead to an increase in hCG free B-subunit levels. ${ }^{13,14}$

Since hCG molecule can be detected in sera and urine specimens of many patients who suffer from cancers, it can be used in following up and predicting long term prognosis of these cancers. According to one study conducted on patients diagnosed with cervical SCC, half of the sample demonstrated immunoreactivity for $\beta$-core hCG in their urine. ${ }^{15}$ Another study revealed hCG free $\beta$-subunit elevation in sera and urine of patients diagnosed with cervical SCC. 8,12

There are also a considerable number of published isolated cases that have shown elevation of hCG in the sera of patients with cervical carcinomas. ${ }^{13,16}$ Norman et $\mathrm{al},{ }^{15}$ showed elevated urinary hCG levels in a small subset of patients diagnosed with cervical intraepithelial

Table 1 - The H-score category numbers and percentages for SCC versus HSIL cases.

\begin{tabular}{lcccccccc}
\hline H-score & \multicolumn{3}{c}{ SCC $(\mathbf{n}=55)$} & \multicolumn{4}{c}{ HSIL $(n=45)$} \\
\hline & \multicolumn{3}{c}{ Percentage score } & \multicolumn{4}{c}{ Percentage score } \\
& 0 & 1 & 2 & 3 & 0 & 1 & 2 & 3 \\
0 & 5 & - & - & - & 7 & - & - & - \\
3 & - & 27 & - & - & - & 38 & - & - \\
6 & - & - & 11 & - & - & - & - & - \\
9 & - & - & - & 12 & - & - & - & - \\
$\mathrm{N}$ & 5 & 27 & 11 & 12 & 7 & 38 & 0 & 0 \\
\hline
\end{tabular}

$\mathrm{H}$-score - histo score, SCC - squamous cell carcinoma, HSIL - high grade squamous intraepithelial lesion. neoplasia and they found elevated urinary hCG levels in $50 \%$ of patients with squamous cell carcinoma.

In our study $84.4 \%$ of HSIL $(n=38 / 45)$ showed diffuse weak positivity, while $15.6 \%$ as $(n=7)$ of them showed no staining with hCG. There was no significant difference to be reported between HSIL areas and the adjacent NSE. On the other hand, 90.9\% ( $n=50 / 55)$ of SCC cases showed strong positivity of hCG, whereas none of the HSIL cases did. The strong reactivity with hCG was significant in the diagnosis of SCC cases ( $p$-value=0.001).

Fenoglio et $\mathrm{al},{ }^{17}$ studied 456 gynecologic cancers and only $18.9 \%$ of SCC showed positive expression of hCG. Meanwhile, Hameed $A,{ }^{18}$ found that there was $52 \%$ hCG immunoreactivity in 63 biopsies of SCC.

Some authors suggested targeting of hCG expression as a medical strategy in managing cancer patients who expressed hCG. In particular, they proposed the possible role of hCG vaccination in treating advanced cervical cancer. These vaccines have the ability of provoking immune response toward hCG which intern, may improve the survival rate of end stage cancer. ${ }^{4,19,20}$

Our study included 55 hysterectomy specimens of SCC revealed $90.9 \%$ hCG immunoactivity which supports the proposed role of hCG in cancer pathogenesis by demonstrating strong hCG immunoreactivity in SCC cases only. The peripheral (epithelial stromal junction) staining in tumor islands in most cases supports this invasion microscopically.

In histology of cervical precancerous and cancerous lesions, it can sometimes be difficult to decide whether there is an invasion, especially in small biopsies, that are not well oriented, or even in some special types of squamous cell carcinoma such as papillary type. Therefore, there should be some strategies to solve this confusion. We suggest that hCG staining can help the pathologists in the diagnosis of such cases, as strong cytoplasmic staining for hCG is a marker of invasive SCC. Cervical biopsies of SCC cases in which the first diagnosis was given, were not examined for hCG expression is a limitation factor for this study.

In conclusion, our study showed hCG expression in SCC cases in paraffin embedded tissues. The expression of hCG compared with HSIL cases. Strong positivity for hCG was detected in $90.9 \%(n=50 / 55)$ of cases whereas $84.4 \%$ of HSIL $(n=38 / 45)$ showed diffuse weak positivity. None of the HSIL cases showed strong positivity. As strong hCG staining is associated with invasion, it can be helpful in challenging cases of histopathologic diagnosis. Further studies conducted on small biopsies with their verified excisional biopsies 
are warranted to establish this staining method as a routine pathology test.

Acknowledgment. We would like to thank EDU Translation for the English language editing.

\section{References}

1. Arbyn M, de Sanjosé S, Saraiya M, Sideri M, Palefsky J, Lacey C, et al. EUROGIN 2011 roadmap on prevention and treatment of HPV-related disease. Int J Cancer 2012; 131 : 1969-1982.

2. Schiffman M, Castle PE, Jeronimo J, Rodriguez AC, Wacholder S. Human papillomavirus and cervical cancer. Lancet 2007; 370: 890-907.

3. Cole LA. hCG, the wonder of today's science. Reprod Biol Endocrinol 2012; 10: 24.

4. Cole LA. HCG variants, the growth factors which drive human malignancies. Am J Cancer Res 2012; 2: 22-35.

5. Iles RK. Ectopic hCGbeta expression by epithelial cancer: malignant behaviour, metastasis and inhibition of tumor cell apoptosis. Mol Cell Endocrinol 2007; 260-262: 264-270.

6. Iles RK, Delves PJ, Butler SA. Does hCG or hCG $\beta$ play a role in cancer cell biology? Mol Cell Endocrinol 2010; 329: 62-70.

7. Butler SA, Iles RK. The free monomeric beta subunit of human chorionic gonadotrophin (hCG beta) and the recently identified homodimeric beta-beta subunit (hCG beta beta) both have autocrine growth effects. Tumour Biol 2004; 25: 18-23.

8. Li D, Wen X, Ghali L, Al-Shalabi FM, Docherty SM, Purkis P, et al. hCG beta expression by cervical squamous carcinoma-in vivo histological association with tumour invasion and apoptosis. Histopathology 2008; 53: 147-155.

9. Burczynska BB, Kobrouly L, Butler SA, Naase M, Iles RK. Novel insights into the expression of CGB1 \& 2 genes by epithelial cancer cell lines secreting ectopic free hCG $\beta$. Anticancer Res 2014; 34: 2239-2248.

10. Burczynska B, Booth MJ, Iles RK, Shah A, Shiled A, Butler SA. SStable knockdown of hCG $\beta$ mRNA expression in bladder cancer cells results in significant growth inhibition. Anticancer Res 2013; 33: 3611-3614.
11. Chang E, LeeE, Oh SJ, Kim JS, Kang C. The immunoexpressions and prognostic significance of inhibin alpha and beta human chorionic gonadotrophins (HCG) in breast carcinomas. Cancer Res Treat 2005; 37: 241-246.

12. Cole LA, Wang YX, Elliott M, Latif M, Chambers JT, Chambers SK, et al. Urinary human chorionic gonadotropin free beta-subunit and beta-core fragment: a new marker of gynecological cancers. Cancer Res 1988; 48: 1356-1360.

13. Mustafa A, Bozdag Z, Tepe NB, Ozcan HC. An unexpected reason for elevated human chorionic gonadotropin in a young woman. Cervical squamous carcinoma. Saudi Med J 2016; 37 : 905-907.

14. Buckner CL, Wilson L, Papadea CN. An unusual cause of elevated serum total beta hCG. Ann Clin Lab Sci 2007; 37: 186-191.

15. Norman RJ, Buck RH, Aktar B, Mayet N, Moodley J. Detection of a small molecular species of human chorionic gonadotropin in the urine of patients with carcinoma of the cervix and cervical intraepithelial neoplasia: comparison with other assays for human chorionic gonadotropin and its fragments. Gynecol Oncol 1990; 37: 254-259.

16. Coleman RL, Lindberg G, Muller CY, Miller DS, Hameed A. Ectopic production and localization of beta-human chorionic gonadotropin in lymphoepithelioma-like carcinoma of the cervix: a case report. Int J Gynecol Pathol 2000; 19: 179-182.

17. Fenoglio CM, Hayata T, Crum CP, Richart RM. The expression of human chorionic gonadotropin in the female genital tract: localization by the immunoperoxidase technique. Diagn Gynecol Obstet 1982;4: 97-103.

18. Hameed A, Miller DS, Muller CY, Coleman RL, AlboresSaavedra J. Frequent expression of beta-human chorionic gonadotropin (beta-hCG) in squamous cell carcinoma of the cervix. Int J Gynecol Pathol 1999; 18: 381-386.

19. Delves PJ, Iles RK, Roitt IM, Lund T. Designing a new generation of anti-hCG vaccines for cancer therapy. Mol Cell Endocrinol 2007; 260-262: 276-281.

20. He LZ, Ramakrishna V, Connolly JE, Wang XT, Smith PA, Jones CL, et al. A novel human cancer vaccine elicits cellular responses to the tumor-associated antigen, human chorionic gonadotropin beta. Clin Cancer Res 2004; 10: 1920-1927. 